\title{
Communicating with accelerated observers in Minkowski spacetime
}

\author{
F J Flores \\ Philosophy Department, California Polytechnic State University, San Luis Obispo, CA 93407, \\ USA \\ E-mail: fflores@calpoly.edu
}

Received 15 June 2007, in final form 31 August 2007

Published 21 November 2007

Online at stacks.iop.org/EJP/29/73

\begin{abstract}
Our goal here is to determine the spatial and temporal constraints on communication between two observers at least one of which moves with constant proper acceleration in two-dimensional Minkowski spacetime. We take as a simplified model of communication one observer bouncing a light signal off another observer. Our derivations use only elementary mathematics and spacetime diagrams, and hence are accessible to students taking their first course in special relativity. Furthermore, the qualitative features of our results can be easily explained to non-physics students in courses that discuss special relativity at a 'conceptual' level.
\end{abstract}

\section{Introduction}

Our aim is to find the special relativistic restrictions on the ability of pairs of observers to communicate with one another when at least one observer is accelerating with constant proper acceleration. We will assume that a pair of observers $O_{1}$ and $O_{2}$ communicates in the following way. $\mathrm{O}_{1}$ sends a light pulse to $\mathrm{O}_{2}$. When $\mathrm{O}_{2}$ receives the light signal, $\mathrm{O}_{2}$ immediately sends a light pulse back to $O_{1}$. Note that as we have described it the relation ' $O_{1}$ communicates with $\mathrm{O}_{2}$ ' is not a symmetrical relation. There are configurations in which, for example, it is possible for $O_{1}$ to communicate with $O_{2}$, but not vice versa. We make no other assumptions about how observers communicate. For example, we do not concern ourselves with the efficiency of this type of communication. There are situations in which, for example, $O_{1}$ will have to wait a very long time indeed for $\mathrm{O}_{2}$ 's reply. Finally, we do not assume that our observers are humans (they clearly need not be), but we do assume that the clocks they carry to measure time along their worldlines are not affected by the accelerations they sustain.

We seek to answer the following two questions for each configuration we consider: (1) 'How far apart (at $t=0$ in the coordinates of a suitably chosen inertial frame) can the two observers be so that communication between them is possible?' and (2) 'For how long (as measured by clocks carried by the observers) can the two observers communicate?' 
First, we consider communication between an inertial observer $I$ and an observer $C$ that moves with constant proper acceleration $a$. I and $C$ are a distance $d$ apart at $t=0$ in the coordinates adapted to $I$ 's inertial rest frame. We show that regardless of the distance $d$ between $I$ and $C$ at $t=0, I$ can communicate with $C$. However, $C$ can communicate with $I$ only if the distance $d$ satisfies $0 \leqslant d<1 / 2 a$. We then show that the proper time $\tau_{I}$ during which $I$ can communicate with $C$ is longer than the proper time $\tau_{C}$ during which $C$ can communicate with $I$. To arrive at this last result requires that we first obtain the Minkowski 'length' of an invariant hyperbola between two points, which we do in the appendix.

Second, we consider communication between two observers $A$ and $B$ that move with the same constant proper acceleration $a$, but are spaced a distance $d$ apart at $t=0$ in the coordinates of a suitably chosen inertial frame. This configuration was first suggested by Dewan and Beran [1] and later discussed by Bell [2]. We find that $A$ can communicate with $B$ only if $d<1 / a$. However, $B$ can communicate with $A$ only if $d<1 / a \phi$, where $\phi$ is the golden ratio. We then show that the proper time $\tau_{A}$ during which $A$ can communicate with $B$ is always longer than the proper time $\tau_{B}$ during which $B$ can communicate with $A$, and we derive the expressions for $\tau_{A}$ and $\tau_{B}$.

Throughout we will use units in which $c=1$. We will use $A, B, C$ and $I$ to designate the worldlines of observers (as opposed to the observers themselves) when the context suffices to fix our meaning. We will also use the familiar fact that the worldline of an observer that moves with constant proper acceleration $a$ along the positive $x$-axis in 2D Minkowski spacetime is an invariant hyperbola with a 'radius of curvature' $\alpha=1 / a$. In general, we can write the equation of motion for such an observer as

$$
(x+\alpha-d)^{2}-t^{2}=\alpha^{2},
$$

where the vertex of the hyperbola is a distance $d$ from the origin of an inertial coordinate system whose $x$-axis coincides with the semi-major axis of the hyperbola (cf [3, pp 73-4]). In the coordinate system in which the origin of the coordinates coincides with the centre of the hyperbola, $d=\alpha$ in (1) and we recover the familiar equation for hyperbolic motion derived in a variety of textbooks ([4, section 2.16], [5, section 14], [6, section 3.7], [7, section 6.2]), namely

$$
x^{2}-t^{2}=\alpha^{2} .
$$

We hope our results will supplement presentations of acceleration in standard relativity textbooks, which are quite varied (see references in [8]). For example, French [9, pp 152-4] focuses on finding the transformation equations for acceleration (as opposed to discussing the notion of proper acceleration), which as he correctly points out is tedious and, in a sense, not very rewarding. Rindler ([4, section 2.16], [5, section 14], [6, section 3.7]) presents a careful discussion of proper acceleration, though he does not consider the specific configurations we do. Finally, Misner, Thorne and Wheeler's discussion [7, section 1-6.3], lying somewhere between the two extremes of French and Rindler, tends to focus on the restrictions on the size of local coordinate systems. We believe that focusing on a few simple cases such as the ones we consider can deepen students' understanding of special relativity.

\section{Constraints on communications between an inertial observer and an accelerated observer}

In this section, we examine the spatial and temporal constraints on communications between an inertial observer $I$ and an accelerated observer $C$. Physically, we can imagine $I$ and $C$ to be initially in a state of relative rest and a distance $d$ apart in the coordinate system $K$ adapted 


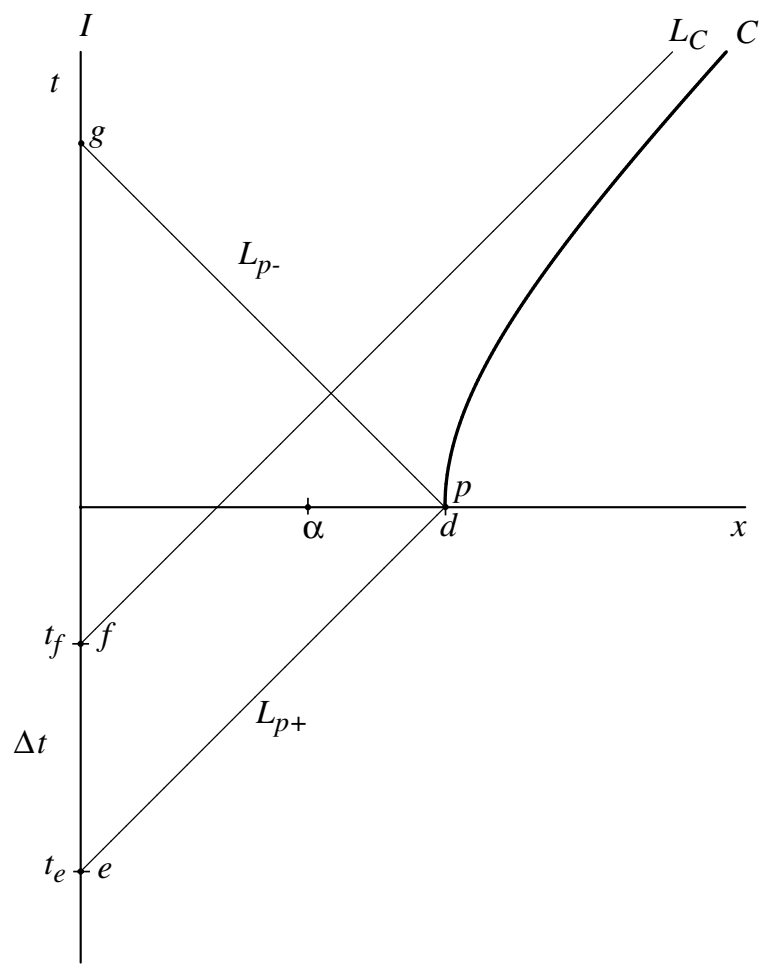

Figure 1. Spacetime diagram displaying that there always exists a time interval $\Delta t$ during which $I$ can communicate with $C$ (shown here for $d>\alpha$ ).

to their mutual rest frame. At $t=0$ (in $K$ coordinates) $C$ receives a light signal from $I$ and begins accelerating with a constant proper acceleration $a$ along the positive $x$-axis. Since we are only interested in communications between $I$ and $C$ once $C$ begins accelerating, we ignore the portion of $C$ 's worldline for times $t<0$. Thus, the equation of motion for $C$ in $K$ is (1), which describes an hyperbola with vertex at $p=(d, 0)$. The equation for the light cone $L_{C}$ that is the asymptote of $C$, which we shall have occasion to use below, is

$$
t=x+\alpha-d .
$$

\subsection{Spatial constraints on communication between I and $C$}

As the spacetime diagram in figure 1 illustrates, for any value of $d$ there exists a time interval $\Delta t$ during which $I$ can communicate with $C$. Regardless of the value of $d$, there exists a light ray $L_{p+}$ that can be emitted by $I$ at the event $e=\left(0, t_{e}\right)$ and that reaches $C$ at the event $p$. There also exists a light ray $L_{p-}$ through $p$ that intersects $I$ at $g$. Thus, $I$ can begin to communicate with $C$ at $e$. $I$ can continue to communicate with $C$ until a time $t_{f}$, which is the time coordinate of an event $f$ on $I$, when the light ray emitted by $I$ happens to be the asymptote $L_{C}$ of $C$. Consequently, there is no spatial constraint on I's ability to communicate with $C$.

The restrictions on the value of $d$ when we consider $C$ communicating with $I$ are different, as one would expect given the asymmetry of the configuration. In order for $C$ to be able to communicate with $I$, a light ray emitted by $C$ at $t \geqslant 0$ and reflected by $I$ must intersect $C$. 


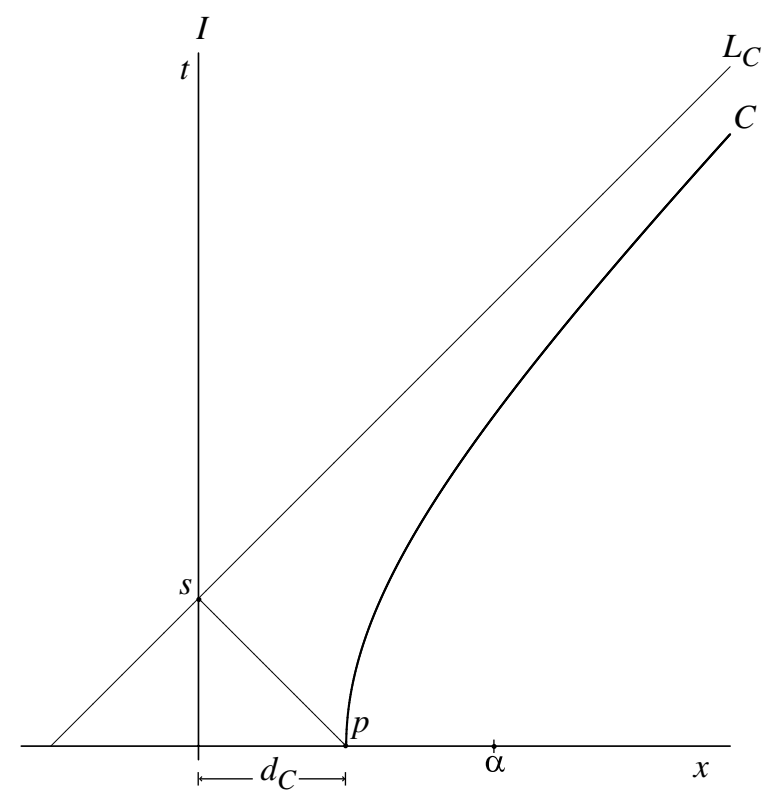

Figure 2. Spacetime diagram displaying that $C$ can communicate with $I$ only if $d<d_{C}$.

The limiting case here occurs when the distance $d_{C}$ between $I$ and $C$ is such that the light ray reflected by $I$ at an event $s$ on $I$ 's worldline is the asymptote $L_{C}$ of $C$ (see figure 2).

When $C$ is at the limiting distance $d_{C}$ from $I$, (3) entails that the coordinates of $s$ are $\left(0, \alpha-d_{C}\right)$ and (1) implies that the coordinates of $p$ are $\left(d_{C}, 0\right)$. Since in this case $s$ and $p$ are connected by the light ray $L_{s p}$, we know that their Minkowski separation has to be zero. We have

$$
d_{C}^{2}-\left(-\alpha+d_{C}\right)^{2}=0 .
$$

Thus, the limiting distance is $d_{C}=\alpha / 2$ and hence $C$ can communicate with $I$ only if $d$ is in the half-open interval $[0, \alpha / 2)$.

\subsection{Temporal constraints on communication between I and $C$}

To determine the proper time $\Delta t=\left.\tau_{I}\right|_{e} ^{f}$ during which $I$ can communicate with $C$, we need only find the $t$-coordinates of the events $e$ and $f$ (see figure 1). We begin by using the point-slope formula to obtain the equation for the light ray $L_{p+}$ :

$$
t=x-d .
$$

We then obtain $t_{e}$ by simply setting $x=0$ in (5) and similarly obtain $t_{f}$ with (3). We find that

$$
\begin{aligned}
& t_{e}=-d \\
& t_{f}=\alpha-d .
\end{aligned}
$$

Since $\Delta t=t_{f}-t_{e}$, we have

$$
\left.\tau_{I}\right|_{e} ^{f}=\alpha .
$$




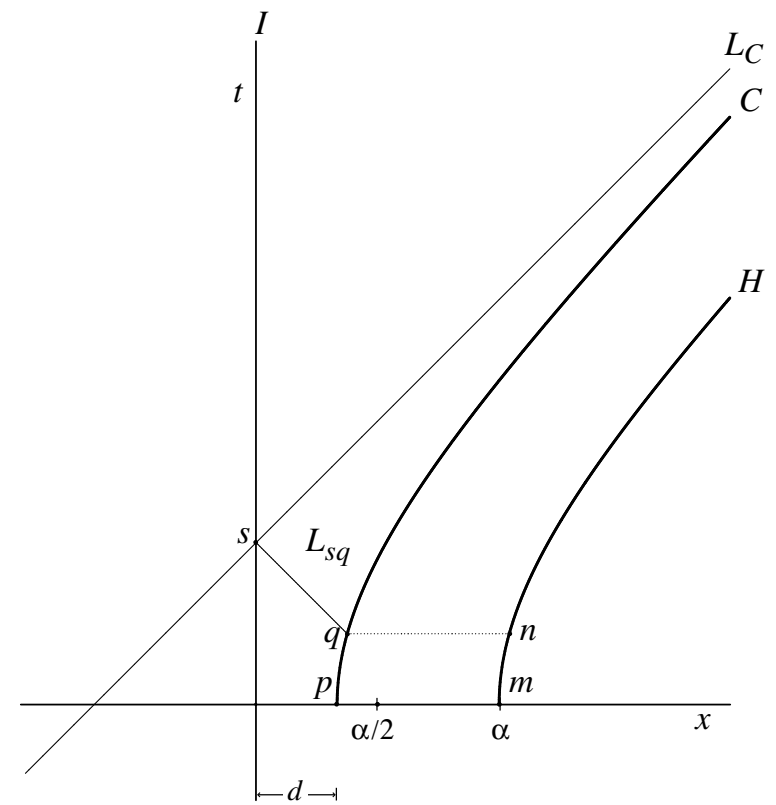

Figure 3. Spacetime diagram displaying that $C$ can communicate with $I$ only between the events $p$ and $q$ (excluding $q$ itself).

Thus, the length of time $\left.\tau_{I}\right|_{e} ^{f}$ during which $I$ can communicate with $C$ is independent of $d$, which we would have expected as we can regard a change in $d$ as a coordinate transformation (i.e., as a translation as opposed to a Lorentz boost).

To determine the time during which $C$ can communicate with $I$, let us consider a case where the distance $d$ between $I$ and $C$ at $t=0$ satisfies $d<\alpha / 2$, as it must so that $C$ can communicate with $I$. As the spacetime diagram in figure 3 illustrates, $C$ can communicate with $I$ only between the events $p$ and $q$ (excluding $q$ itself). Our goal is thus to find $\left.\tau_{C}\right|_{p} ^{q}$. However, $\left.\tau_{C}\right|_{p} ^{q}=\left.\tau_{H}\right|_{m} ^{n}$, where $m=(\alpha, 0)$ is the vertex of an invariant hyperbola $H$ with radius of curvature $\alpha$ and $n$ is the image of $q$ we obtain by projecting $q$ parallel to the $x$-axis onto $H$ (see figure 3). Thus, we can find $\left.\tau_{C}\right|_{p} ^{q}$ by finding the coordinates of $n$ and using (A.3), which gives the expression for $\left.\tau_{H}\right|_{m} ^{n}$ in terms of $x_{n}$ and $t_{n}$.

To find the coordinates of $n$, we first find the coordinates of $q$ and then perform the following translation:

$$
\begin{aligned}
& x_{n}=x_{q}+(\alpha-d) \\
& t_{n}=t_{q} .
\end{aligned}
$$

We find the coordinates of $q$ by finding the equation for the light ray $L_{s q}$ and finding its intersection with $C$. From (3) it follows directly that $s=(0, \alpha-d)$. Since the slope of $L_{s q}$ is -1 and $L_{s q}$ goes through the point $s$, we can use the point-slope formula to obtain the equation for $L_{s q}$ :

$$
t=-x+\alpha-d .
$$

Solving for the intersection of (11) and (1), we find 


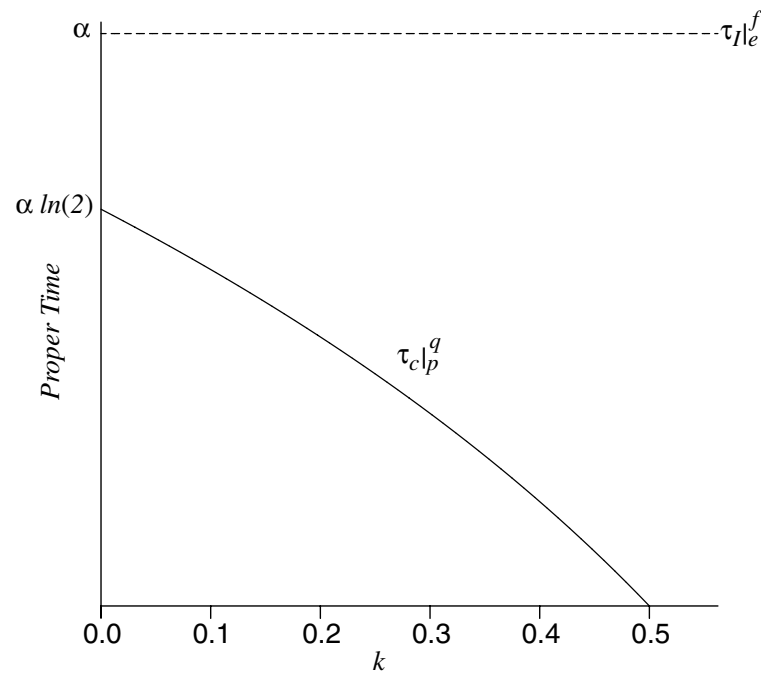

Figure 4. Graph of proper time $\left.\tau_{I}\right|_{e} ^{f}$ during which $I$ can communicate with $C$ and proper time $\left.\tau_{C}\right|_{p} ^{q}$ during which $C$ can communicate with $I$ as a function of $k$, where the distance $d$ between $I$ and $C$ is $d=k \alpha$.

$$
\begin{aligned}
& x_{q}=\left(\frac{1}{4}\right)\left(\frac{\alpha^{2}}{\alpha-d}\right) \\
& t_{q}=\left(\frac{1}{4}\right)\left(\frac{3 \alpha^{2}-8 \alpha d+4 d^{2}}{\alpha-d}\right) .
\end{aligned}
$$

Using (9) and (10), we obtain

$$
\begin{aligned}
& x_{n}=\left(\frac{1}{4}\right)\left(\frac{5 \alpha^{2}-8 \alpha d+4 d^{2}}{\alpha-d}\right) \\
& t_{n}=\left(\frac{1}{4}\right)\left(\frac{3 \alpha^{2}-8 \alpha d+4 d^{2}}{\alpha-d}\right) .
\end{aligned}
$$

With the aid of (A.3) and using (14) and (15), we find that

$$
\left.\tau_{C}\right|_{p} ^{q}=\alpha \ln \left(\frac{2(\alpha-d)}{\alpha}\right) .
$$

Finally, if we let $k=d / \alpha$, which in this case will satisfy $0 \leqslant k<1 / 2$, we have

$$
\left.\tau_{C}\right|_{p} ^{q}=\alpha \ln (2(1-k))
$$

Thus, $C$ can communicate with $I$ for an amount of proper time $\left.\tau_{C}\right|_{p} ^{q}$ that is always less than the amount of proper time $\left.\tau_{I}\right|_{e} ^{f}$ during which $I$ can communicate with $C$ as we illustrate in figure 4.

\section{Constraints on communications between two accelerated observers}

We now focus on the spatial and temporal constraints on communication between two observers $A$ and $B$ both of which move with constant proper acceleration $a$. We imagine that $A$ and $B$ 


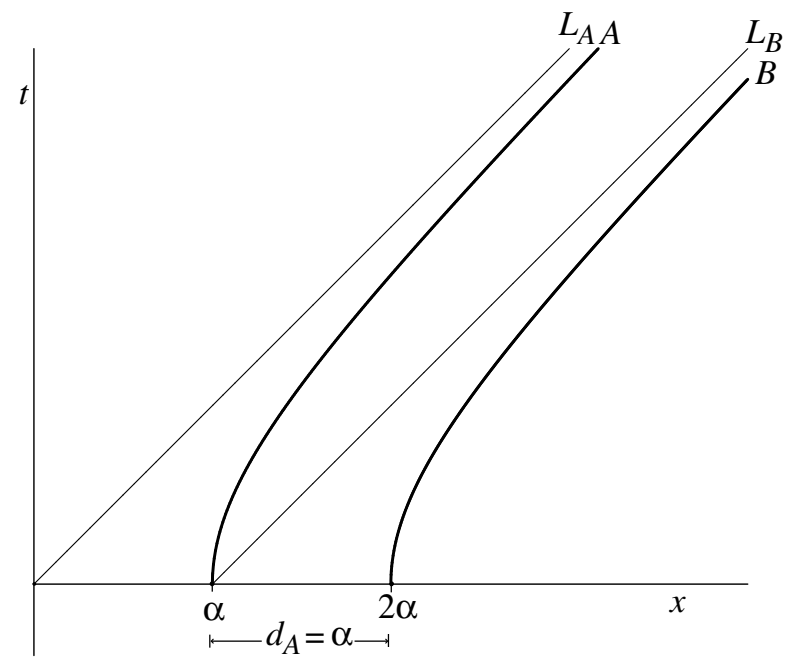

Figure 5. Spacetime diagram displaying that $A$ cannot communicate with $B$ if $d_{A} \geqslant \alpha$.

begin accelerating when they are a distance $d$ apart at $t=0$ in the inertial coordinate system $K$. For simplicity, we choose $K$ so that its $x$-axis coincides with the semi-major axis of $A$ and $B$, and so that the origin of $K$ coincides with the centre of the hyperbola $A$.

Physically, we can imagine $A$ and $B$ to be originally in a state of relative rest until they each receive a light ray from an observer that is equidistant from $A$ and $B$ in $K$. $A$ and $B$ then begin accelerating along the positive $x$-axis with the same constant proper acceleration $a$. As before, since we are only interested in communication between $A$ and $B$ once $A$ and $B$ begin accelerating, we ignore the worldlines of both $A$ and $B$ for times $t<0$.

\subsection{Spatial constraints on communications between $A$ and $B$}

An elementary spacetime diagram (see figure 5) illustrates that there exists a maximum distance $d_{A}=\alpha$ such that if $d \geqslant d_{A}$ then $A$ cannot communicate with $B$. If $d \geqslant d_{A}$, no light ray leaving $A$ ever reaches $B$, which is just an illustration of the familiar result that if a photon begins a distance $d \geqslant 1 / a$ 'behind' an observer that moves with constant proper acceleration $a$, the photon can never catch up to the observer. Thus, $A$ can communicate with $B$ only if $0<d<d_{A}$. We say that $d>0$, because if $d<0$ the roles of $A$ and $B$ are simply reversed. The case where $d=0$ is the trivial case where the worldlines of $A$ and $B$ are coincident.

The spatial limitations on communications from $B$ to $A$ are different. In order for $B$ to receive a light ray reflected off $A$, the light ray must be emitted by $B$ at a coordinate time $t \geqslant 0$ and the reflected light ray must intersect with (the worldline of) $B$. The limiting case here occurs at a coordinate distance $d_{B}$ when the light ray reflected by $A$ just happens to be the asymptote $L_{B}$ of $B$.

We can obtain the value of $d_{B}$ with the aid of the spacetime diagram in figure 6 by first finding the equation of the light ray $L_{s q}$, where $s$ is now the point of intersection of the light ray through $q$ with negative slope and the $x$-axis. We then impose the additional constraint that the $x$-coordinate of $s$ is $\alpha+d_{B}$ to find $d_{B}$ in terms of $\alpha$. 


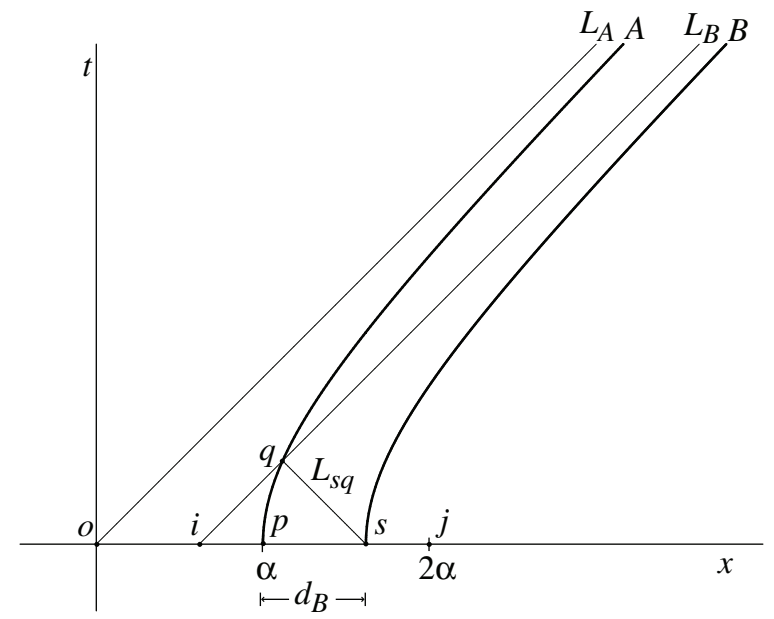

Figure 6. Spacetime diagram displaying that $B$ can communicate with $A$ only if $d<d_{B}$.

In the coordinate system $K$, the equation of motion for $A$ is (2). The equation of motion for $B$ is

$$
(x-d)^{2}-t^{2}=\alpha^{2},
$$

where $d$ is now the distance between $A$ and $B$ at $t=0$. The equations for the light rays $L_{A}$ and $L_{B}$ that are the asymptotes to the hyperbolae $A$ and $B$, respectively, are simply

$$
\begin{aligned}
& x=t \\
& x=t+d .
\end{aligned}
$$

The coordinates of $q$, which we obtain by finding the intersection of (2) and (20), are

$$
\begin{aligned}
& x_{q}=\left(\frac{1}{2}\right)\left(\frac{\alpha^{2}}{d}+d\right) \\
& t_{q}=\left(\frac{1}{2}\right)\left(\frac{\alpha^{2}}{d}-d\right) .
\end{aligned}
$$

Finally, using the coordinates of $q$, and the fact that the slope of $L_{s q}$ is -1 , we obtain the following equation for the light ray $L_{s q}$ :

$$
t=-x+\frac{\alpha^{2}}{d} \text {. }
$$

In the particular case where the distance between $A$ and $B$ is $d_{B}$, the equation of the light ray $L_{s q}$ is simply

$$
t=-x+\frac{\alpha^{2}}{d_{B}}
$$

Setting $t=0$ in (24) we see that the $x$-coordinate of $s$ is

$$
x_{s}=\frac{\alpha^{2}}{d_{B}} \text {. }
$$




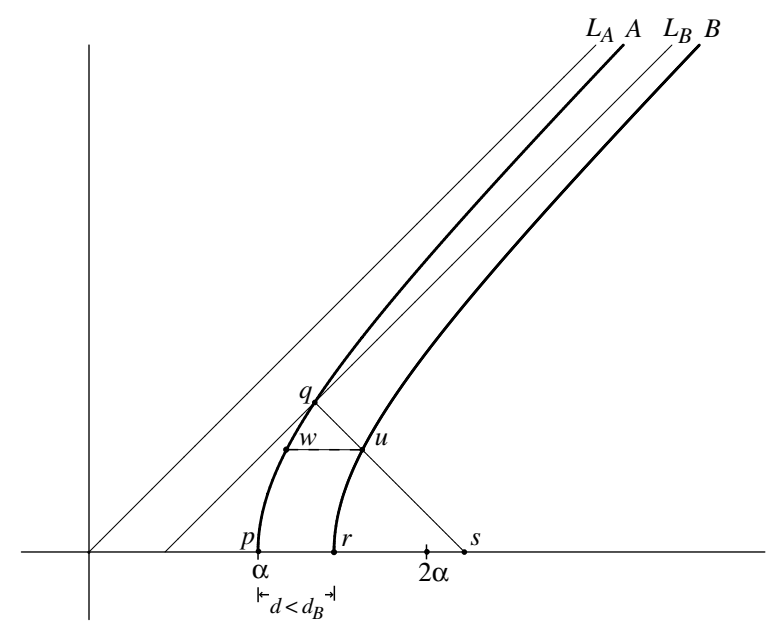

Figure 7. Spacetime diagram displaying constraints on communication for two observers $A$ and $B$ that move with the same constant proper acceleration $a$ and are spaced apart a distance $d<d_{B}$ in $K$.

Furthermore, we also know that the $x$-coordinate of $s$ is

$$
x_{s}=\alpha+d_{B} .
$$

Using (25) and (26), we find that

$$
d_{B}=\frac{1}{2}(\sqrt{5}-1) \alpha .
$$

However, as we have already seen, $d_{A}=\alpha$. Consequently, we have

$$
d_{B}=\frac{1}{2}(\sqrt{5}-1) d_{A} .
$$

The expression for $d_{B}$ in terms of $d_{A}$ does not seem very 'pretty' until we note that the numerical constant in (28) is simply $\frac{1}{\phi}=(\phi-1)$, where $\phi$ is the golden ratio. Thus, $B$ can communicate with $A$ only if the distance between the two observers at $t=0$ in $K$ is $d_{B}<d_{A} / \phi$. Furthermore, suppose we let $o$ be the origin of $K, i$ the $x$-intercept of $L_{B}, p$ the $x$-intercept of $A$ and $j$ be the point with coordinates $(2 \alpha, 0)$, the latter being of interest because it is a distance $d_{A}$ from $p$ (see figure 6). The Minkowski lengths (which we indicate with vertical bars) of the following line segments are in the golden ratio:

$$
\frac{|\overline{p j}|}{|\overline{p s}|}=\frac{|\overline{o p}|}{|\overline{o i}|}=\phi \text {. }
$$

Finally, we note that since the lengths in (29) are Minkowski intervals, (29) is an invariant relation.

Suppose now that the distance between the two observers $A$ and $B$ (at $t=0$ in $K$ ) is $d<d_{B}$, as it must be in order for both observers to be able to communicate with one another. A simple spacetime diagram (see figure 7) shows that there exists a pair of events $p$ and $q$ on $A$ such that $A$ can communicate with $B$ only during events on $A$ 's worldline that are between $p$ and $q$ (excluding $q$ itself).

Similarly, there exists a pair of events $r$ and $u$ such that $B$ can communicate with $A$ only during events on $B$ 's worldline that are between $r$ and $u$ (excluding $u$ itself). Furthermore, since the worldline of $B$ is the same curve as the worldline of $A$ only shifted along the positive $x$-axis by a distance $d<d_{B}$, the spacetime diagram shows that the amount of proper time 
during which $B$ can communicate with $A$ is shorter than the proper time during which $A$ can communicate with $B$.

\subsection{Temporal constraints on communication between $A$ and $B$}

We wish now to find the proper times $\left.\tau_{A}\right|_{p} ^{q}$ and $\left.\tau_{B}\right|_{r} ^{u}$ to compare the proper time during which $A$ can communicate with $B$ with the proper time during which $B$ can communicate with $A$. We obtain $\left.\tau_{A}\right|_{p} ^{q}$ by substituting the coordinates of $q$ from (21) and (22) into (A.3) to obtain

$$
\left.\tau_{A}\right|_{p} ^{q}=\alpha \ln \left(\frac{\alpha}{d}\right)
$$

To find $\left.\tau_{B}\right|_{r} ^{u}$, we first note that $\left.\tau_{B}\right|_{r} ^{u}=\left.\tau_{A}\right|_{p} ^{w}$, where $w$ is the event on $A$ that we obtain by projecting $u$ parallel to the $x$-axis onto $A$ (see figure 7). Thus, we can use (A.3) to find the expression for $\left.\tau_{B}\right|_{r} ^{u}$ if we know the coordinates of $w$. We obtain the latter by first finding the coordinates of $u$ from the intersection of $L_{s q}$ and $B$, i.e., of (23) and (18). We then perform a simple translation to shift the $x$-coordinate of $u$ by a distance $d$ (toward the origin) to obtain the coordinates of $w$ :

$$
\begin{aligned}
& x_{w}=\left(-\frac{1}{2}\right)\left(\frac{\alpha^{4}-\alpha^{2} d^{2}+d^{4}}{d\left(d^{2}-\alpha^{2}\right)}\right) \\
& t_{w}=\left(-\frac{1}{2}\right)\left(\frac{\alpha^{4}-3 \alpha^{2} d^{2}+d^{4}}{d\left(d^{2}-\alpha^{2}\right)}\right) .
\end{aligned}
$$

Finally, using (31) and (32) in (A.3), we find

$$
\left.\tau_{B}\right|_{r} ^{u}=\alpha \ln \left(\frac{\alpha^{2}-d^{2}}{\alpha d}\right) .
$$

To compare the proper times $\left.\tau_{A}\right|_{p} ^{q}$ and $\left.\tau_{B}\right|_{r} ^{u}$ it is useful once again, as in section 2.2, to express them in terms of $k=d / \alpha$. Thus, we have

$$
\begin{aligned}
& \left.\tau_{A}\right|_{p} ^{q}=\alpha \ln \left(\frac{1}{k}\right) \\
& \left.\tau_{B}\right|_{r} ^{u}=\alpha \ln \left(\frac{1-k^{2}}{k}\right) .
\end{aligned}
$$

A graph of $\left.\tau_{A}\right|_{p} ^{q}$ and $\left.\tau_{B}\right|_{r} ^{u}$ as functions of $k$ for a fixed value of $\alpha$, such as the one depicted in figure 8 , gives us a good picture of how these proper times compare.

Finally, to emphasize the beautiful role that $\phi$ plays in the geometry, note that when $k=\frac{1}{\phi}$, we have

$$
\left.\tau_{B}\right|_{r} ^{u}=\alpha \ln \left(\frac{1-\frac{1}{\phi^{2}}}{\frac{1}{\phi}}\right) .
$$

However, since $\phi$ satisfies the relation $\frac{1}{\phi}=\phi-1$, (36) becomes

$$
\left.\tau_{B}\right|_{r} ^{u}=\alpha \ln (1)
$$

And hence, when $k=\frac{1}{\phi},\left.\tau_{B}\right|_{r} ^{u}=0$. 


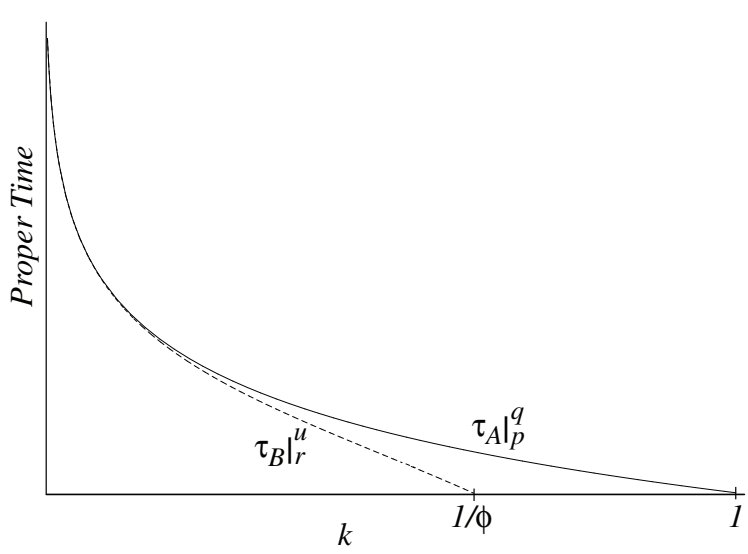

Figure 8. Comparison of proper times during which $A$ can communicate with $B$ and vice versa for a fixed value of $\alpha$ as a function of $k$, where the distance $d$ between $A$ and $B$ is $d=k \alpha$.

\section{Conclusion}

The results we have derived concerning the spatial and temporal constraints on communication between two observers at least one of which moves with constant proper acceleration can be adapted to emphasize other important 'conceptual' lessons. For example, the discussion concerning the limits on communication between $I$ and $C$ can be a useful prelude to discussions concerning communication with an observer that falls into a black hole (such as one can consider in the Schwarzchild solution in general relativity). Similarly, the discussion concerning the limits on communication between $C$ and $I$ can also help introduce a discussion (of the sort provided by Misner, Thorne and Wheeler [7, pp 163-9]) concerning the limitations on the size of a local coordinate system. Finally, we hope the concrete examples we discuss can enhance students' understanding of the subtle analogy between acceleration in Newtonian mechanics and proper acceleration in special relativity.

\section{Acknowledgment}

I would like to thank an anonymous referee for valuable suggestions on how to improve this paper. I would also like to thank O T Fernflores for stimulating discussions especially concerning spaceship B.

\section{Appendix. Minkowski 'length’ of an invariant hyperbola}

Using the expression for the Minkowski metric, we find the expression for the Minkowski 'length' $l_{G}$ of an arbitrary worldline $G$ between the events $a$ and $b$ (which is just the proper time $\left.\tau_{G}\right|_{a} ^{b}$ between $a$ and $b$ ):

$$
\left.l_{G}\right|_{a} ^{b}=\int_{x_{a}}^{x_{b}} \sqrt{\left(\frac{\mathrm{d} t}{\mathrm{~d} x}\right)^{2}-1} \mathrm{~d} x .
$$

We now wish to find the proper time between a pair of events $m$ and $n$ along the invariant hyperbola $H$ with radius of curvature $\alpha=1 / a$ and vertex $(\alpha, 0)$. We focus on finding the expression for the proper time along $H$, because the hyperbola $A$ we consider in the text is 
coincident with $H$; the hyperbolae $B$ and $C$ in the text can be made to coincide with $H$ by performing a simple translation. Thus, we aim to find the Minkowski length $\left.\tau_{H}\right|_{m} ^{n}$ of $H$ from the vertex of the hyperbola $m=(\alpha, 0)$ to an arbitrary point $n=\left(x_{n}, t_{n}\right)$ on $H$.

Differentiating (2) and substituting into (A.1) leads to the standard integral in (A.2), which with aid of (2) yields (A.3):

$$
\begin{aligned}
\left.\tau_{H}\right|_{m} ^{n} & =\int_{\alpha}^{x_{n}} \frac{\alpha}{\sqrt{x^{2}-\alpha^{2}}} \mathrm{~d} x \\
\left.\tau_{H}\right|_{m} ^{n} & =\alpha \ln \left(\frac{x_{n}+t_{n}}{\alpha}\right) .
\end{aligned}
$$

\section{References}

[1] Dewan E and Beran M 1959 Note on stress effects due to relativistic contraction Am. J. Phys. 27 517-8

[2] Bell J S 1993 How to teach special relativity Speakable and Unspeakable in Quantum Mechanics (Cambridge: Cambridge University Press) pp 67-80

[3] Møller C 1972 The Theory of Relativity (Oxford: Oxford University Press)

[4] Rindler W 1977 Essential Relativity (Berlin: Springer)

[5] Rindler W 1991 Introduction to Special Relativity 2nd edn (Oxford: Oxford University Press)

[6] Rindler W 2001 Relativity: Special, General, and Cosmological (Oxford: Oxford University Press)

[7] Misner C, Thorne K S and Wheeler J A 1973 Gravitation (San Francisco: Freeman)

[8] Tartaglia A and Ruggiero M L 2003 Lorentz contraction and accelerated systems Eur. J. Phys. 24 215-20

[9] French A P 1968 Special Relativity (New York: Norton) 